Vol. 3, No. 1 | January - June 2019

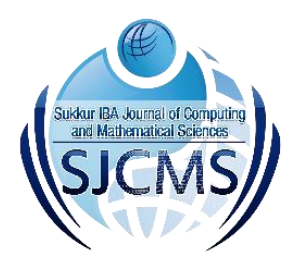

\title{
Robotic Techniques Used for Increasing Improvement Rate in The Rehabilitation Process of Upper Limb Stroke Patients
}

\author{
Faheem Ahmed ${ }^{1}$, Aqeel Ahmed ${ }^{1}$, Zeeshan Bhatti ${ }^{1}$
}

\begin{abstract}
:
The rate of stroke patients in Pakistan is increasing, resulting in the decrease mobility of the patients. The movement of the upper limb stoke patient is decreased due to the weakness and loss of joint control in his upper body. To improve the coordination of movement of the upper limb stroke patients, many methods e.g. passive and active modes for improving the disrupted mobility are introduced. The objectives of this paper are to first review the studies on upper limb stroke patients and the techniques used for increasing the improvement rate through physical therapy by exoskeleton and evaluation of the performance of the patient using methods such as quantification and graphical representations so that it can be shown to the patient for his motivation to improve further. The paper introduces a mechanical design of exoskeleton with 1 degree of freedom for elbow and 2 degrees of freedom for shoulder movement for rehabilitation of joints of stoke patients. It also mentions the safety that will be taken in the process so that the exoskeleton is safe to use when it is in contact with humans. The model of the exoskeleton has the characteristic of being modular and easily operable and use admittance control strategy. The control strategy of the exoskeleton is discussed to maintain the position and orientation of the device and also is able to cater the gravitational attraction which plays an important part in the movement of the actuators. The mathematical model of motion attained due to the degrees of freedom of the exoskeleton is then evaluated and the lastly areas where the future work of exoskeleton can be done are discussed.
\end{abstract}

Keywords: Rehabilitation, Exoskeleton, robotic techniques, admittance control, physical therapy, coordinatedmovement, stroke patients, 3 degrees of freedom, mechanical design

\section{Introduction}

In the field of rehabilitation medicine, a physiotherapist is working for the better muscle movement of stroke and orthopedic patients. There is a need for physical exercise on a regular basis so that the patient is able to recover quickly. According to A Sunderland, D J Tinson et al., the studies have shown that the patient is able to recover quickly if he gets encouragement and is able to move his body muscles in homebased exercises and use of bio-feedback therapy [1].

The patient feels that he is a burden on his family and the society. This is true as there is a cost of living and welfare of the patient, which reduces his motivation and also decrease the motor outcomes [2]. If he is provided a good nursing, hospital care and good assistance at home, he is able to recover quickly and assist his family monetarily [2].

Upper limb injury in stroke and orthopedic patients is a common problem and it does require rehabilitation. The injury not only affects the patient, but also affects his family economically as he is not earning and is dependent on others which creates further consequences if his recovery takes a long time [3]. The study done by Roberto Colombo, Fabrizio Pisano et al states that if we

\footnotetext{
${ }^{1}$ Institute of Information and Communication Technology, University of Sindh, Jamshoro Corresponding Email: faheemas1907@ gmail.com
} 
want to optimize the motor outcome of the patient then we have to evaluate the patient's rehabilitation in a quantitative way and also show those results to the patients. This way the patients will get motivated and will try to achieve more quantitative motor outcome value, resulting in shorter rehabilitation time. [2].

Considering Low Income Countries (LIC) like Pakistan, the rehabilitation of stroke and orthopedic patients is performed mostly manually. This increases high personal cost of the physiotherapist, the time and money invested by the family to go to the rehabilitation center will also increase. Basic kind of exercises can be performed at home, but due to lack of knowledge, the patient is not able to perform the same exercises at home for recovery, which creates frustration and decreases the motivation of the patient [3].

Some devices that are used by the physiotherapists uses a continuous passive mode of operation, which means that the robot does not take any feedback and is performing the same tasks within a regular interval of time and limits. These kinds of devices are not used for diagnosis as there are no sensors integrated in it [3].

Due to its versatile applications of robotics, this branch of engineering is growing its importance every day. It provides numerous opportunities to the patients and to the physiotherapists in increasing the rehabilitation outcomes and lowering the cost of therapy [4].

To broadly classify rehabilitation robots, two categories are used: end effector and exoskeletons.

End-effector rehabilitation robots: End effort is the distal part of the robot which is attached to the patient's limb and is used for the movement to his body. For Example, MIT-Manus and ARM Guide [5].

Exoskeleton rehabilitation robots: These robots have at least two points of contact. It allows torque at individual body joints. The mapping is used to align the joint of exoskeleton with the joints of the user. Examples of Exoskeleton are mpower ARM Brace, ARMin III, Rice wrist and CADEN 7 [5].

Exoskeleton imitates the external structural mechanism of a human body with its corresponding joints and links. These devices have high torque actuators installed in the exoskeletal body to assist the human in its routine tasks. These devices are growing in their popularity due to their use in the field of rehabilitation medicine and virtual reality i.e., It is beneficial for disabled people and also for healthy population [6].
The exoskeleton has a different mode of operations designed with different control algorithms to assist them in their routine tasks. The uses of the exoskeleton are as follow:

1. Rehabilitation Medicine (Physiotherapy): It has a great application in the physical therapy of patients as it helps in the training exercises of the patient to increase the extremity of the joints of the patient.

2. Assistive Devices: These devices work as human amplifier. It interacts with the objects that are in its surroundings by carrying most of the load. The operator do not have to worry about the load carried by the body.

3. Haptic and Master/Slave Devices: The exoskeleton is present in virtual or real environment and the interaction it has with its environment are transmitted to the user as a feedback. The user is able to feel the conveying interaction of the exoskeleton by knowing the shape, texture, stiffness and other characteristics of that environment depending on the sensors installed in the device. This mode is used in simulation as well as tele-operations.

The exoskeleton has a great application in the field of medicine. The robotic aided therapy's effectiveness is gaining its popularity among the patients with spinal cord injury and stroke. The main purpose that these devices serve in conducting rehabilitation, improving the outcomes of patient and quantifying the improvement of the patients through rehabilitation research and methodologies. Some techniques that are used to quantify the improvements include range of motion (ROM), smoothness in movement and strength. These values are used for the feedback to the exoskeleton if it is working in active mode or can be utilized by the therapist and researchers in robot design and control [5].

One of the best-known exoskeleton rehabilitation robots is InMotionARM Interactive Therapy System is continuously taking data from the patients and is trying to challenge the patient to improve his motor therapy. The other one that is similar to InMotion ARM Interactive Therapy System is MAHI EXO II. It has a definite workspace and the torque output and bandwidth to match with the human capabilities. Low inertia, static friction and other damping also result in improved control system [5]. 
To measure the progress of patients, a research was conducted by Janet H. Carr, Roberta B. Shepherd to measure the motor capabilities was introduced known as Motor Assessment Scale (MAS). The quantitative analysis through this medium was found to be highly reliable in clinical practice. The progress of each patient was easily analyzed in later cases and the score achieved by the patient also boost up the motivation scale of the patient to rehabilitate earlier [7].

\section{Problems in Pakistan}

The rehabilitation in Low Income Countries (LIC) including Pakistan is a bit different. According to research conducted in Pakistan Institute of Prosthetic and Orthotic Sciences (PIPOS), there are over 30 million individuals in LICs who require prosthetic and orthotic services. The percentage of Upper limbs surpass the percentage of Lower limb therapy to a great extent [8].

The responsibilities of physiotherapists in LIC are greater as the patients do not want to depend on the expensive treatment from the machines. The rehabilitation process is itself time consuming and also very expensive [9]. The patients do not feel comfortable when asked for a large amount for health services. Economic problems arise in these countries to get the required funding in rehabilitation projects and to get the required training from High Income Countries (HIC) to Low Income Countries (LIC).

So, there is a need of making technology locally that is economical, easily available and easy maintained. As the device is made locally, proper training to the technicians can be arranged easily. In Pakistan, Research in the field of rehabilitation on a larger scale is very limited. Some of the institutes working in Pakistan are DOW University of Health and Sciences, Liaquat National Hospital, Dept. of PTR, Hayatabad Paraplegic Centre and National Institute of Rehabilitation Medicine. But the output of these institutes should be increased so the patients are able to once again take the burden of their family and give back to the society.

\section{Model Design}

There are many techniques which are used for the rehabilitation of stroke patients. Some of them are Electrical Stimulation, Physical Therapy, Passive Manipulation and sensory Motor Rehabilitation Technique [2]. Electrical Stimulation and Passive Manipulation techniques are used in early stages of therapy when there is no movement in the limbs of the patients. Once the signals in the body are restored, then it is time for the physical or active therapy to rehabilitate further movement of the limbs. Our exoskeleton model focuses on physical therapy using sensory motor rehabilitation techniques.

According to Johanna $\mathrm{H}$ van der Lee et al, in clinical messages, intensive exercise therapy for the rehabilitation of stroke patients appears to be beneficial in their rapid recovery. The improvement is also dependent on how much the patients are putting effort in exercise. Therefore, the patient should be encouraged time to time to exercise more often so that he rehabilitates quickly [10].

A Motor Assessment Scale (MAS) model was created during 1980s by Janet H. Carr and Roberta B. Shepherd to quantify the rehabilitation of the patient [7]. The aims of the project were to monitor the daily activities of the patient display the result in a quantified manner which is understandable by other health professionals. The best performance of the patient should also be displayed for the motivation of the patients to perform well in the rehabilitation process. As it was a software-based model therefore it was also inexpensive to implement in a rehabilitation machine.

While modelling the design of exoskeleton, similar strategies were considered as explained below:

\section{Mathematical Model}

The exoskeleton model involves three degrees of freedom. Two for shoulder joint and one for elbow joint each incorporating a high torque motor. Through simplification, the closed loop of a motor can be related to a first order damp system which means that the control system has no overshoot.

A simplified diagram of one arm of exoskeleton is shown in figure 1 . 


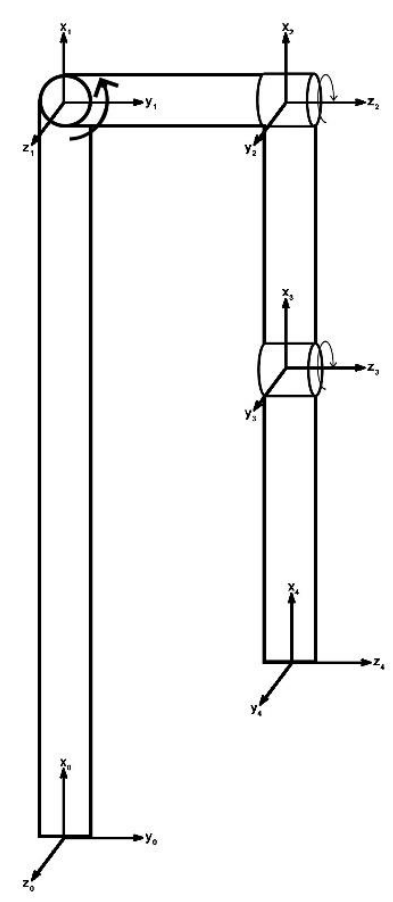

Figure 1: One arm of exoskeleton

Table I: DH parameters for the exoskeleton

\begin{tabular}{|c|c|c|c|c|}
\hline & $\theta i$ & $d i$ & $A i$ & $\alpha i$ \\
\hline 1 & 0 & 10 & 0 & 0 \\
\hline 2 & $\theta_{1}$ & 0 & 2 & 90 \\
\hline 3 & $\theta_{2}$ & -3 & 0 & 0 \\
\hline 4 & $\theta_{3}$ & -3 & 0 & 0 \\
\hline
\end{tabular}

Using the values given in Table $\mathrm{I}$, the homogeneous transformation of each step is calculated as:

$$
\begin{aligned}
A_{2}= & {\left[\begin{array}{cccc}
c_{1} & 0 & s_{1} & 2 c_{1} \\
s_{1} & 0 & c_{1} & 2 s_{1} \\
0 & 1 & 0 & 0 \\
0 & 0 & 0 & 1
\end{array}\right] } \\
A_{3}= & {\left[\begin{array}{cccc}
c_{2} & -s_{2} & 0 & 0 \\
s_{2} & c_{2} & 0 & 0 \\
0 & 0 & 1 & -3 \\
0 & 0 & 0 & 1
\end{array}\right] } \\
A_{4}= & {\left[\begin{array}{cccc}
c_{3} & -s_{3} & 0 & 0 \\
s_{3} & c_{3} & 0 & 0 \\
0 & 0 & 1 & -3 \\
0 & 0 & 0 & 1
\end{array}\right] }
\end{aligned}
$$

Using these values, the rotation and transformation matrices for the end-effector are:

$R=\left[\begin{array}{ccc}c_{1} c_{2} c_{3}-c_{1} s_{2} s_{3} & -c_{1} s_{2} c_{3}-c_{1} c_{2} s_{3} & c_{1} \\ s_{1} c_{2} c_{3}-s_{1} s_{2} s_{3} & -s_{1} s_{2} c_{3}-s_{1} c_{2} s_{3} & c_{1} \\ \left.s_{2}+s_{3}\right) & c_{2}+c_{3} & 1\end{array}\right]$

$T=\left[\begin{array}{c}2 c_{1}-6 s_{1} \\ 2 s_{1}-6 c_{1} \\ 10\end{array}\right]$

Using these values from the DH-parameter, the simulation in MATLAB showed following result showing all the three links on one side from the base of the exoskeleton.

$$
A_{1}=\left[\begin{array}{cccc}
1 & 0 & 0 & 0 \\
0 & 1 & 0 & 0 \\
0 & 0 & 1 & 10 \\
0 & 0 & 0 & 1
\end{array}\right]
$$




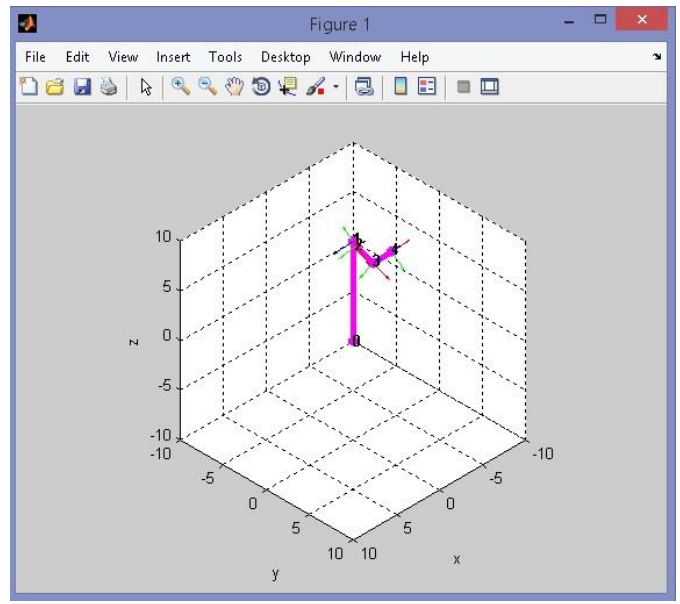

Figure 2: Tree links on same side of Exoskeleton.

\section{Hardware Design}

The exoskeleton comprises of three parts. The manipulator mechanical moving structure, electrical part - electrical drives, actuators and power circuitry and the computer - for controlling the exoskeleton. While considering hardware design, the following conditions were taken into consideration:

The body of the exoskeleton should be made of non-conductive durable material. The body of the exoskeleton can be made using Laser cutting (light weight steel, carbon fiber or aluminum) or using $\mathrm{CNC}$ machines for fabrication. Easy to wear and removing straps were also part of patient's facilitation so use of Velcro was necessary. The links of exoskeleton with foam/cotton pads be used to remove blood blockage of the patient and increase comfortability.

The structure should be mechanically well, easy to manufacture and easier for maintenance purposes. The design of the exoskeleton should include adjustable height and shoulder sizes.

Great care should be taken on the links that are in contact with the patient. The designer has to first define the maximum and the minimum rotation of the joints. If the exoskeleton exceeds its rotational motion, it might become a liability and will affect the rehabilitation process. Limiter switched should be incorporated in the design for maxima and minima so that the motors should not go beyond the required limits.
Joints of stroke are stiff and resist motion as there is no blood flow in their arteries and veins. The same is the case with orthopedic patients, after the operation, the arm is at rest for 1-2 months, reducing the moving capability of the arm thus the muscles get stiff. To cater this problem, high torque servo motors or linear actuators can be implemented in the design to overcome the resistance provided by patients. A controller should be integrated to operate the motors of exoskeleton, gets necessary feedback from the actuators and decides the path to reach its destination point. To compensate weight, friction and spatial gravity, the motors in stall position will require power to provide the required amount of compensation torque [11]. For the protection of the actuators, casing should be used.

At the initial stage of rehabilitation, PID controller cannot be used as our requirement is not reaching to the destination point quickly, rather than we want to gradually increase the motor capability of the patient. Once the motor capacity is increased, a PIC controller can be implemented for fast to and fro motion to reduce the stiffness in the joints. Gain (Kp, Ki and $\mathrm{Kd}$ ) of PIC controller can be controlled to control the speed of the motor rotation.

Microcontroller will be controlling the electrical components of the exoskeleton for controlling the overall circuitry of the hardware.

A regulated power supply circuitry is needed to reduce the hazard of increasing current limits while the motor is working on high torque. Safety consideration like kill switches and fuses should also be taken while designing the electrical circuitry to save the patient in case exoskeleton misbehave.

Due to unknown environment, the inaccuracies due to round-off errors will increase and can be calculated using:

$$
\begin{gathered}
\text { Robot accuracy }=1 / 2(\text { Basic Resolution Unit })+ \\
\text { Mechanical Accuracy }
\end{gathered}
$$

\section{Control Design}

According to Johanna $\mathrm{H}$ van der Lee, et al. the control system must be of simple design and should integrate the methods which involve effective therapy to improve the movement of the stroke patients [10]. 
There are two modes in which rehabilitation of stroke patient is done. Passive mode (Impedance Control) exercises and active mode (Admittance Control) exercises.

In passive mode exercises, the patient is not able to move his/her arm, the exoskeleton will support the arm and will help the patient to exercise. It means the displacement is being input from the therapist and the motor motion is the output for the exercise of the patient [2].

While in active mode exercises, the patient has recovered some movement and has a spatial area in which he is able to move his arm but lacks the coordination. In this method, the exoskeleton should know what the recovered areas of the patient are and assist the patient in those areas where he/she finds difficult to move his muscles [11]. It means, the force is applied by the patient and the movement of exoskeleton is the output. This method will help the patient to move his muscles a little further and recover some more ground to speed up his/her recovery process.

To improve the shoulder-elbow coordination, the program is easier to understand and use accordingly. After a therapy from the exoskeleton, the improvements of the patients should be monitored. The therapist will guide the program depending on the performance of the patient i.e. the need of active mode exercises or passive mode exercises. As the patient learns a certain degree of the motor learning program, the level of difficulty will increase, thus making it challenging for the patient and increasing the motor movement in less amount of time [11].

This could be achieved through Adaptive-assistance-as-needed algorithm. This algorithm utilizes a combination of feedback as well as feedforward loops. The feedback loop is controlled by the position of the motors of exoskeleton that how much the motors are actuated while the feedforward loop depends on the ability of the patients to improve his/her joints without assistance of the exoskeleton. As the feedforward loop increases, the feedback loop will decrease. It means greater the patient tries to achieve his/her recovery objectives to accomplish a motor task, the dependency on the exoskeleton will reduce. This decrease in feedback loop will show the better recovery of the patient [11].

Games play a vital part in catching the attention of the user and improve the coordination of brain with the body. It is a great source of learning and can be implemented for rehabilitation. The idea behind implementing games in admittance control is by using the feedback from the exoskeleton, a virtual 3D space is designed. In that space, obstacles will be placed near the locality of the patient's motor outcomes. The task of the patient is to go beyond the limits of his joint movement to achieve those tasks thus enabling him in faster recovery.

A simple technique for taking feed forward control is by using EEG and ECG signals from the wrist and elbow of the patient, but this has transmission interference due to sweat from patients and poor signal to noise ratio (S/N ratio) [11]. This system can be improved by implementing and algorithm parallel to ECG and EEG which calculates the movement of patient without assistance from exoskeleton using the position feedback of from servo motors and the reduce drainage of power during this time and provide the compensation in the error cause by EEG and ECG.

\section{Result}

Using the above analysis, a 3D model of exoskeleton was modelled in SolidWorks. The maxima and minima were examined. All the parameters discussed above, were implemented in the model.

To make the size of the exoskeleton modular for each patient, prismatic joints are used for the base so that it is easier for the therapist to adjust the size for each patient as shown in the figure 3.

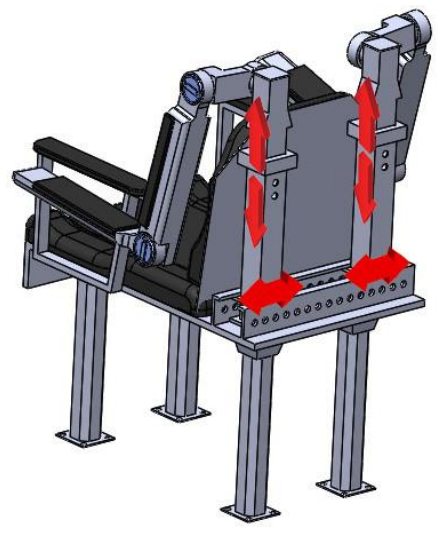

Figure 3: Adjustable prismatic joints 
The motion of each revolute joint was monitored for maxima and minima and how much they are able to rotate in free space so that they can be controlled using the described control strategy as illustrated in figure 4.

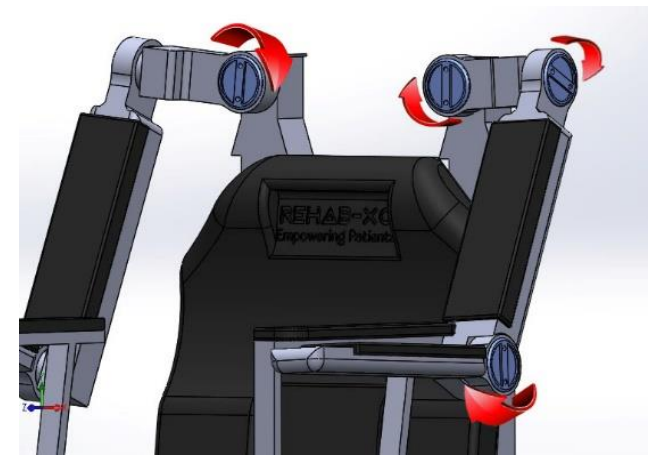

Figure 4: Rotation of each revolute joints.

\section{Conclusion}

In this research work, a technique used in robotics was used for assisting stroke patients with their rehabilitation and physiotherapy, explicitly the upper limb, with a special designed chair. The model is easy to fabricate and is a platform for implementing our admittance control algorithm for faster rehabilitation of stroke and orthopedic patients. In order to improve the motivating level of the patient, path orientation games can be used in control design so that the patient can move his/her joints to move to the desired location in space in order to achieve the task of the game.

\section{REFERENCES}

[1]. A Sunderland, D J Tinson et al. (1992). Enhanced physical therapy improves recovery of arm function after stroke. A randomised controlled trial. J NeurolNeurosurg Psychiatry 1992 55: pp 530-535.

[2]. Roberto Colombo, Fabrizio Pisano et al (September 2005). Robotic Techniques for Upper Limb Evaluation and Rehabilitation of Stroke Patients, IEEE TRANSACTIONS ON NEURAL SYSTEMS AND REHABILITATION ENGINEERING, VOL. 13, NO. 3, SEPTEMBER 2005, pp 311-324

[3]. Andreas Wege, Konstantin Kondak, and Gunter Hommel (July 2005). Mechanical Design and Motion Control of a Hand Exoskeleton for Rehabilitation; Proceedings of the
IEEE International Conference on Mechatronics \& Automation, Niagara Falls, Canada. July 2005, pp 155-159.

[4]. Ali UtkuPehlivan, OzkanCelik, Marcia K. O’Malley (July 2011). Mechanical Design of a Distal Arm Exoskeleton for Stroke and Spinal Cord Injury Rehabilitation; 2011 IEEE International Conference on Rehabilitation Robotics, Rehab Week Zurich, ETH Zurich Science City, Switzerland, June 29 - July 1, 2011.

[5]. James A. French, Chad G. Rose, Marcia K. O’Malley (October, 2014). SYSTEM CHARACTERIZATION OF MAHI EXO-II: A ROBOTIC EXOSKELETON FOR UPPER EXTREMITY REHABILITATION; Proceedings of the ASME 2014 Dynamic Systems and Control Conference, San Antonio, TX, USA, October 22-24, 2014.

[6]. Joel C. Perry, Jacob Rosen (AUGUST 2007). Upper-Limb Powered Exoskeleton Design; IEEE/ASME TRANSACTIONS ON MECHATRONICS, VOL. 12, NO. 4, AUGUST 2007 pp 408-417

[7]. JANET H. CARR, ROBERTA B. SHEPHERD, (February 1985). Investigation of a New Motor Assessment Scale for Stroke Patients. PHYSICAL THERAPY; Volume 65 / Number 2, February 1985 pp175-180.

[8]. Fahad Moazzam Dar, Daniyal Malik, et al (March 2013). Automation of Prosthetic upper limbs for transhumeral amputees using switch-controlled motors, The International Journal of Soft Computing and Software Engineering [JSCSE], Vol. 3, No. 3, pp 593-599

[9]. Aziz Ahmad, (2009). Prosthetic problem with transbial amputee, JPMI 2009 Vol: 23, No. 2: 153-158.

[10]. Johanna $H$ van der Lee, Ingrid AK Snels, et al (30th September 2000). Exercise therapy for arm function in stroke patients: a systematic review of randomized controlled trials. Clinical Rehabilitation 2001; 1 5: pp 2031.

[11]. NathanaëlJarrassé, TommasoProietti et al. (01 Dec 2014). Robotic exoskeletons: a perspective for the rehabilitation of arm coordination in stroke patients, Frontiers in Human Neuroscience, December 2014, Volume 8, Article 947 pp 1-13. 